\title{
Tailoring Kidney Transport of Organic Dyes with Low Molecular-weight PEGylation
}

Bujie Du†, Xingya Jiang $\dagger$, Yingyu Huang†, Siqing Li†, Jason C Lin $\dagger$, Mengxiao Yu† and Jie Zheng*†

† Department of Chemistry and Biochemistry, The University of Texas at Dallas, Richardson, Texas, 75080, United States

Corresponding Authors *E-mail: jiezheng@utdallas.edu

\section{Content}

Figure S1. Synthesis and chemical structure of 800CW-PEGn

Figure S2. Mobility of 800CW-PEGn in agarose gel

Figure S3. UV-vis absorption and fluorescence spectra of 800CW-PEGn

Figure S4. Protein binding test of 800CW-PEGn

Scheme S1. The method to quantify the renal clearance efficiency of organic dyes.

Figure S5. $k 12$ of $800 \mathrm{CW}-\mathrm{PEGn}$

Figure S6. Pharmacokinetics parameters of 800CW-PEGn

Figure S7. The 800CW-PEG45 distribution in the kidneys at $1 \mathrm{~h}$ post injection

Figure S8. k12 and k21 of 800CW-PEGn

Figure S9. Pharmacokinetics of ZW800-1 and ZW800-1-PEG45

Figure S10. Pharmacokinetics of FL-PEG22 and FL-PEG45

Figure S11. The relationship between molecular weight with hydrodynamic radius of folded molecule (PEG) and unfolded protein

Figure S12. The comparison among 800CW-PEG45, ZW800-1-PEG45 and fluorescine-PEG45 in elimination rate constant (kel). 
a) IRDye 800CW NHS Ester

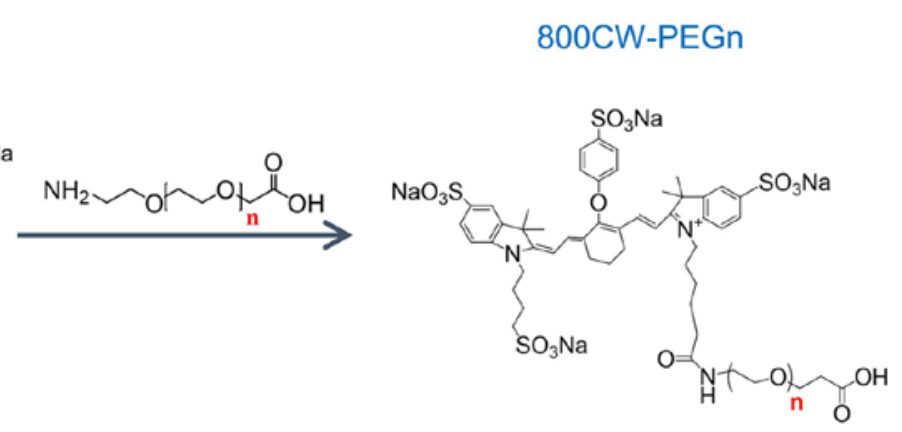

b)
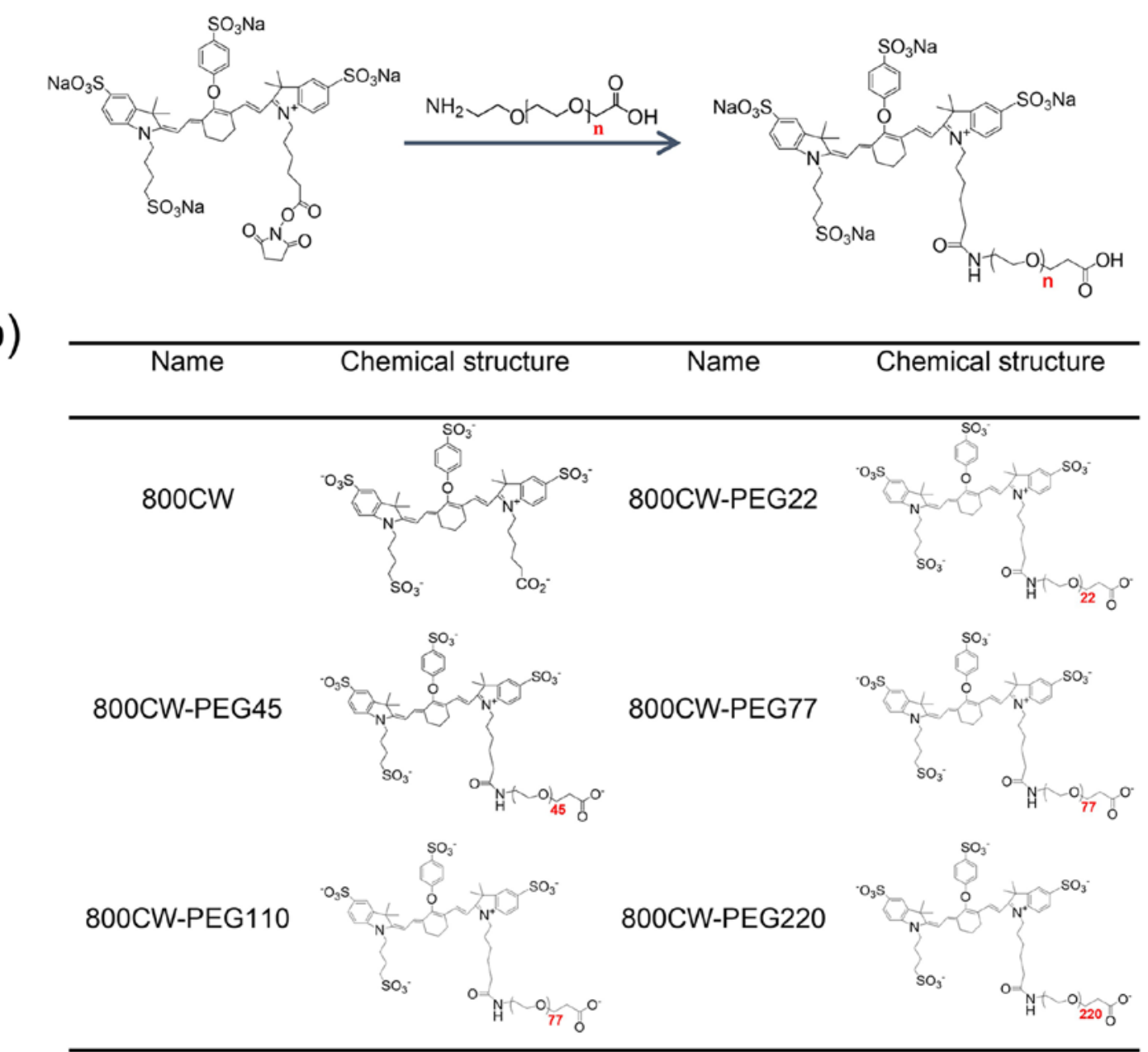

Figure S1. Synthesis of 800CW-PEGn (a) and chemical structures of 800CW, 800CWPEG22, 800CW-PEG45, 800CW-PEG77, 800CW-PEG110 and 800CW-PEG220 (b). 


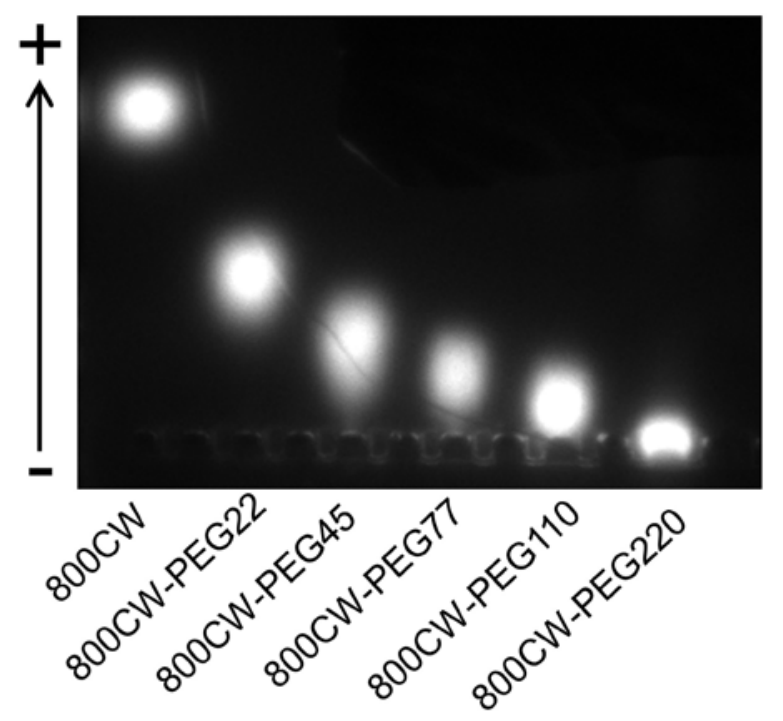

Figure S2. Mobility of 800CW-PEG conjugates in 2\% agarose gel. High molecular weight conjugates move slower than low molecular weight ones.
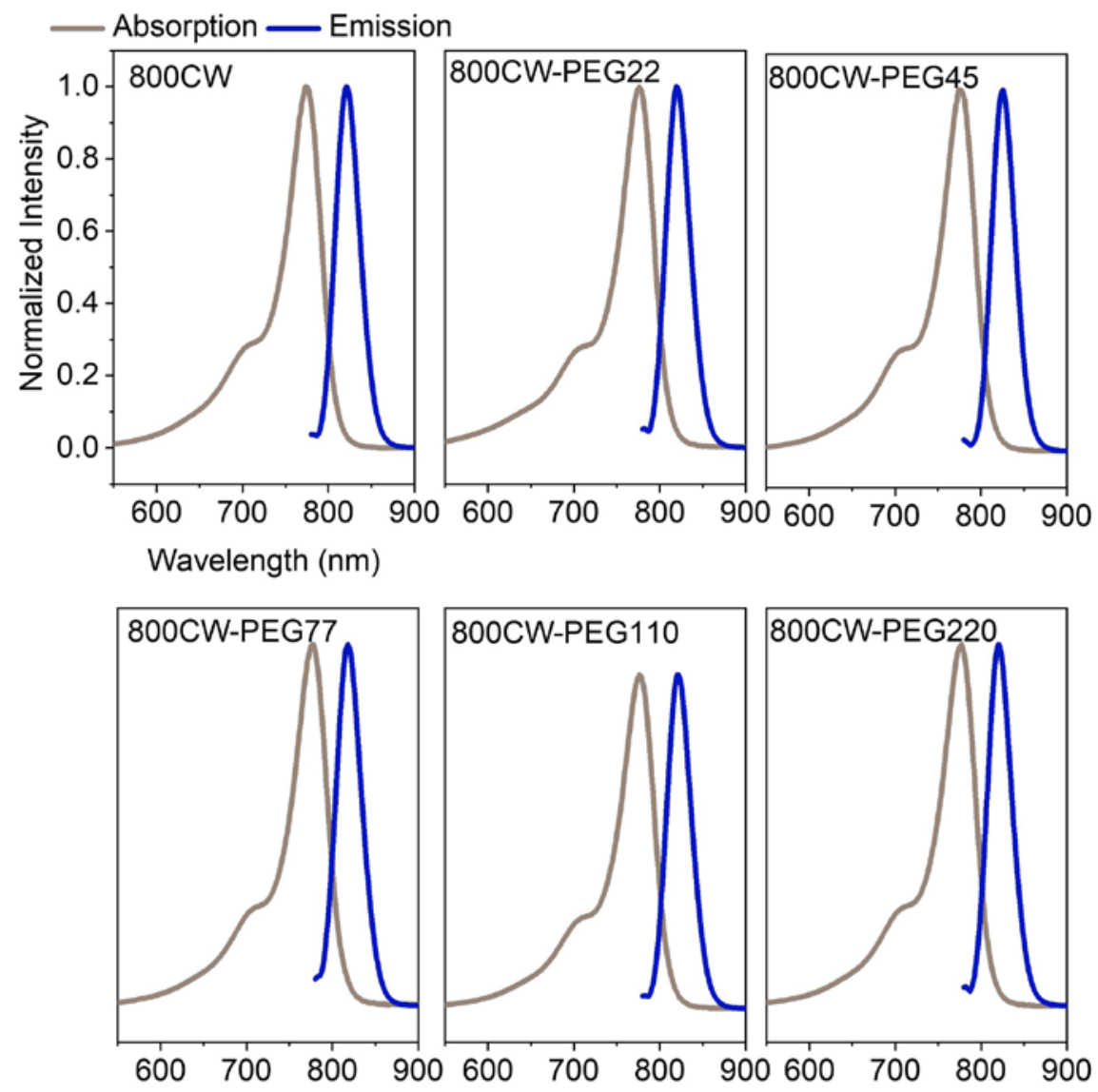

Figure S3. UV-vis absorption and fluorescence spectra of 800CW-PEGn. 
a)
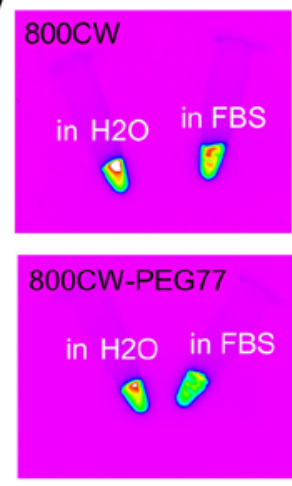
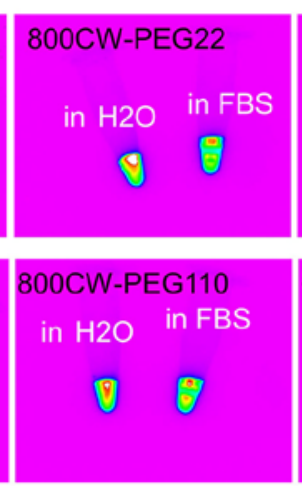
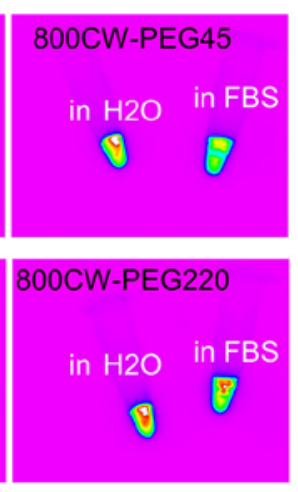

b)

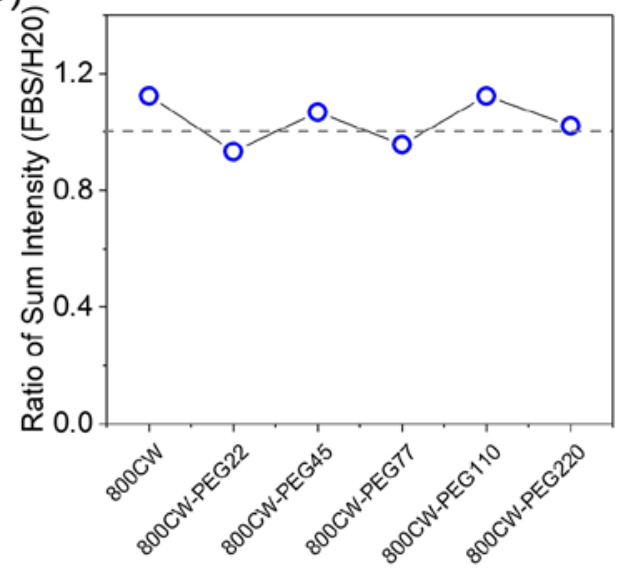

Figure S4. Protein binding test of $800 \mathrm{CW}$ and $800 \mathrm{CW}-\mathrm{PEG}$ by quantifying their fluorescence intensity change in water and in serum. a) Fluorescent images of 800CW and 800CW-PEG conjugates in water and in 100\% fetal bovine serum (FBS) (same dye concentration). b) The ratio of sum intensity in FBS over that in water of 800CW and 800CW-PEG conjugates. All values of ratio were near 1 , indicating that both $800 \mathrm{CW}$ and $800 \mathrm{CW}-\mathrm{PEG}$ conjugates resisted protein binding.

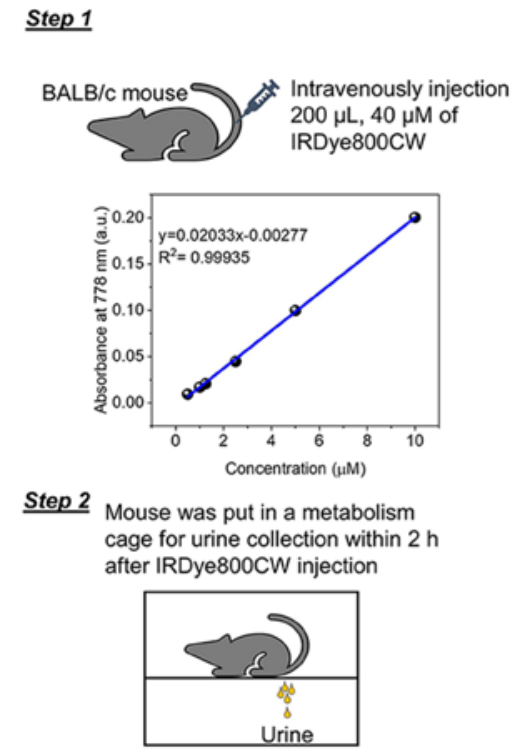

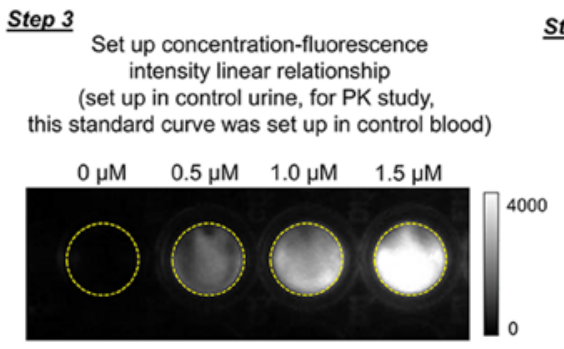

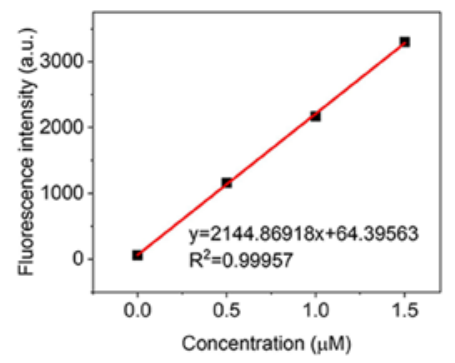

$\underline{\text { Step } 4}$

Collect urine at $2 \mathrm{~h}$ post injection and measure its fluorescence intensity under same condition with standard curve setting up

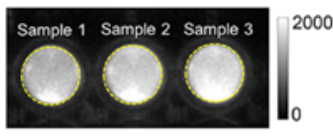

\section{$\underline{\text { Step } 5}$}

Calculation: based on the standard curve set up in step 3, we convert the fluorescence intensity to concentration of IRDye $800 \mathrm{CW}$ in urine and then calculated the renal clearance efficiency of IRDye800CW at $2 \mathrm{~h}$ with unit of \% ID based on the injection dose.

* The renal clearance of other dye and conjugates were measured with same way as IRDye800CW

** The PK studies of all dyes and conjugates were quantified in similar with the quantification of renal clearance

Scheme S1. The method to quantify the renal clearance efficiency of IRDye800CW. The blue curve is the linear relationship between the concentration of IRDye800CW in PBS and the absorbance intensity at $778 \mathrm{~nm}$. The injection dosage is quantified based on this blue standard curve. The red curve is the linear relationship between concentration of IRDye800CW in urine and its intensity at $790 \mathrm{~nm}$ under the excitation of $710 \mathrm{~nm}$. 


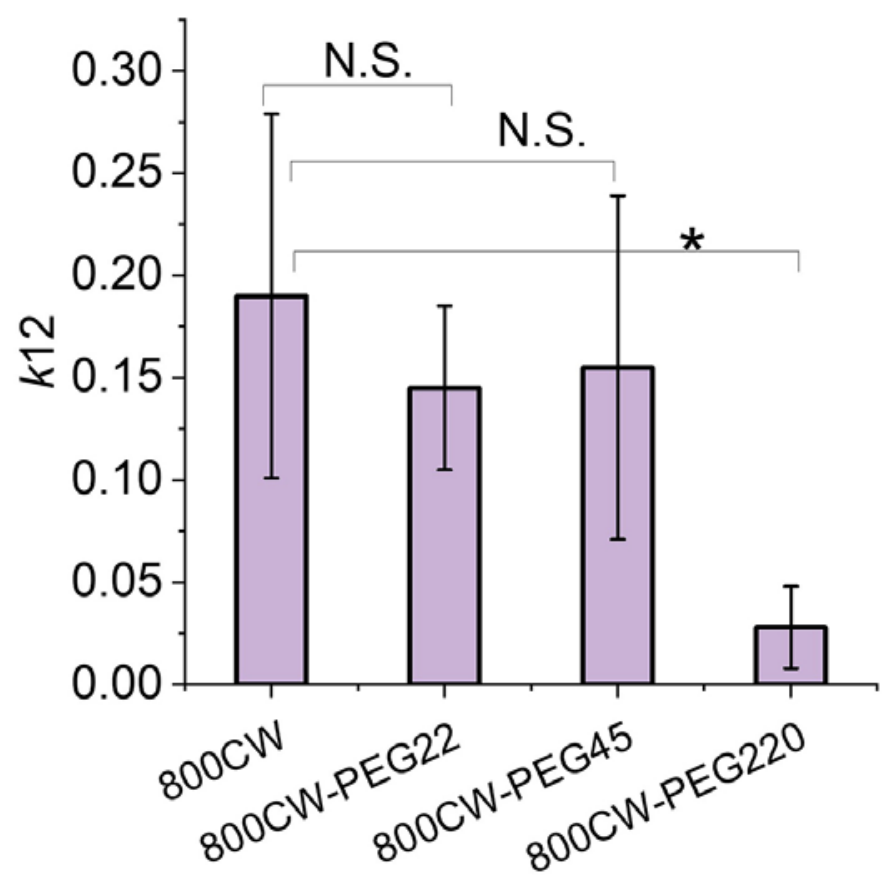

Figure S5. The $k 12$ of 800CW wasn't influenced by conjugation of PEG22 and PEG45 but was reduced by conjugation of PEG220. $\mathrm{n}=3$. ${ }^{*} \mathrm{P}<0.05$, N.S. $\mathrm{P}>0.05$, Student's t-test. 
a)

c)
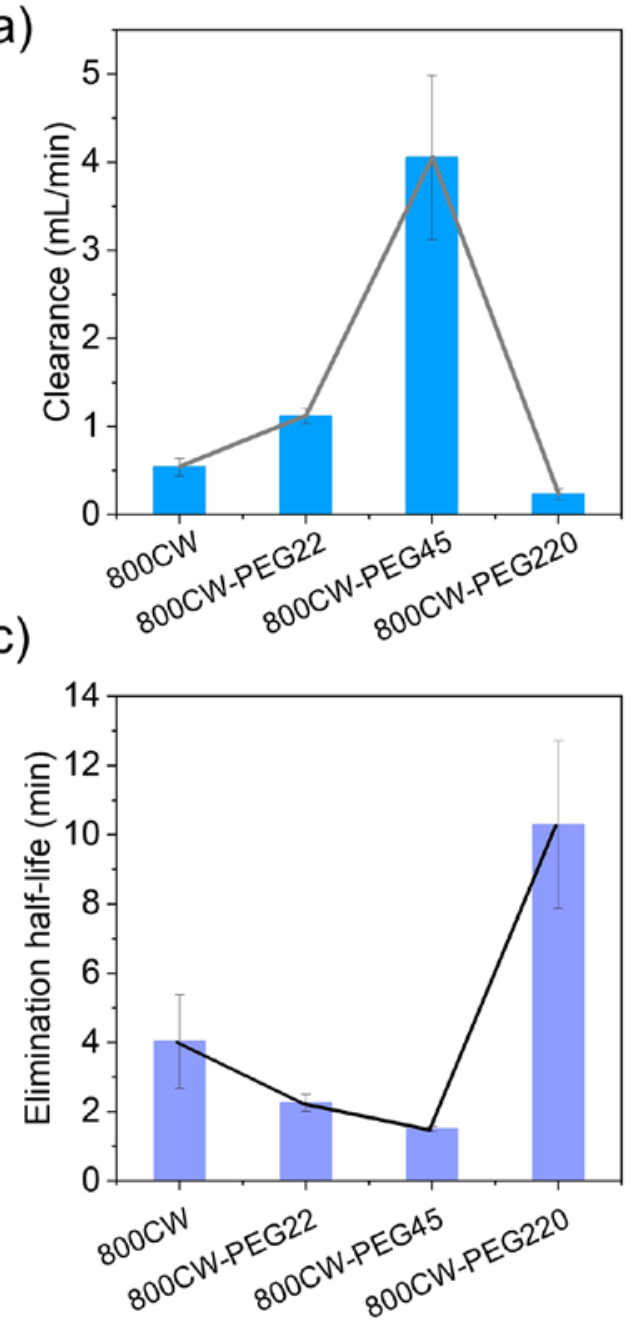

b)
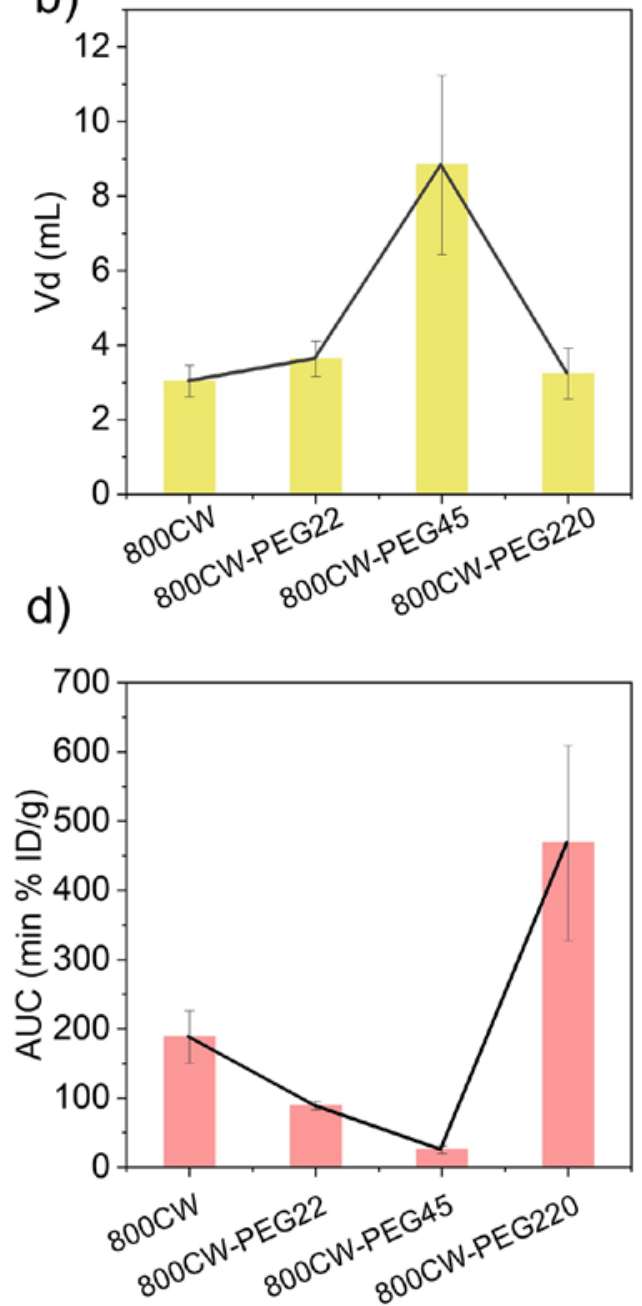

Figure S6. Pharmacokinetics parameters of 800CW-PEGn. a) The clearance value of $800 \mathrm{CW}$, 800CW-PEG22, 800CW-PEG45 and 800CW-PEG220. b) The volume of distribution (Vd) of 800CW, 800CW-PEG22, 800CW-PEG45 and 800CW-PEG220. c) The elimination half-life of 800CW, 800CW-PEG22, 800CW-PEG45 and 800CW-PEG220. d) The area under the curve (AUC) of 800CW, 800CW-PEG22, 800CW-PEG45 and 800CW-PEG220. Clearly, the 800CW-PEG45 behaves as the turning point among 800CW-PEGn in terms of these four parameters. The enhanced clearance of 800CW-PEG45 resulted in its shorter elimination halflife and smaller AUC than free 800CW. 


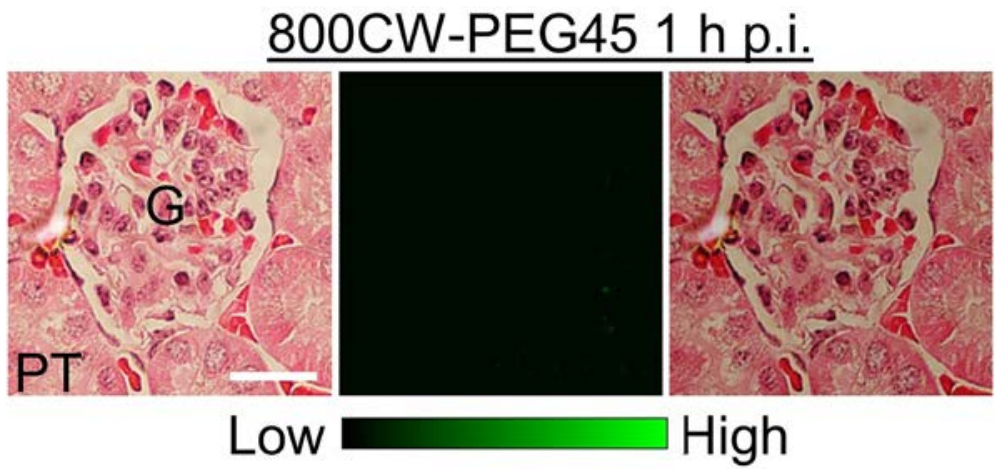

Figure S7. Fluorescence images of glomerulus and tubules at tissue level at $1 \mathrm{~h}$ post injection of 800CW-PEG45 (same color scale bar in Figure 3d). G, glomerulus, Kidney tissue was stained by Hematoxylin and Eosin (H\&E) stain. Fluorescence images were taken at 720/790 nm. Scalar bar is $20 \mu \mathrm{m}$.

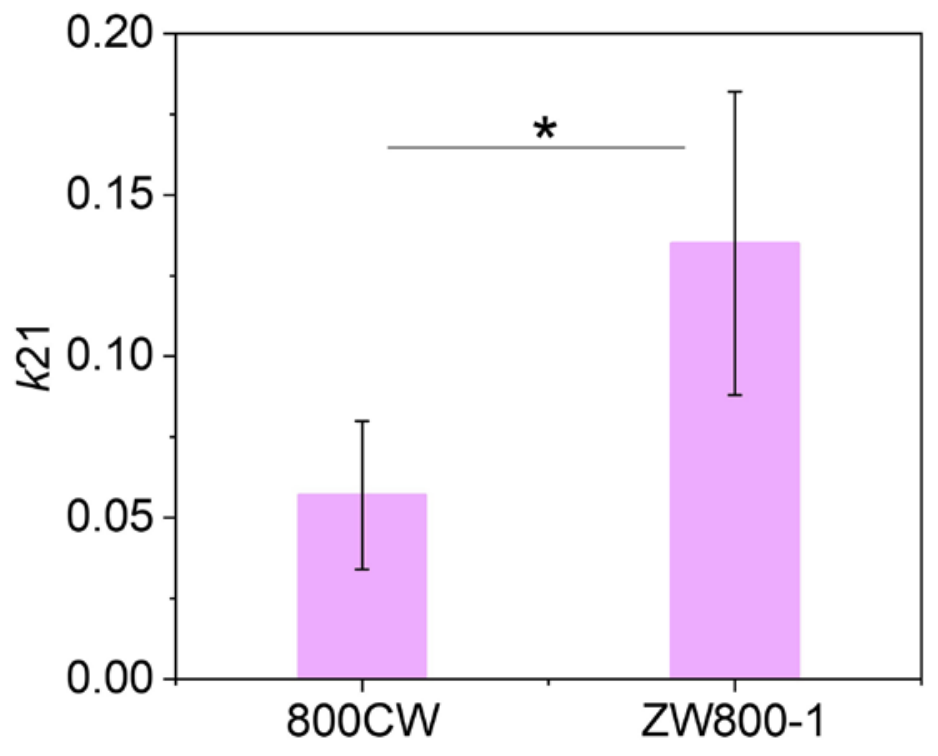

Figure S8. The comparison of $k 21$ between IRDye800CW (800CW) and ZW800-1. n=3. $* \mathrm{P}<0.05$, Student's t-test 


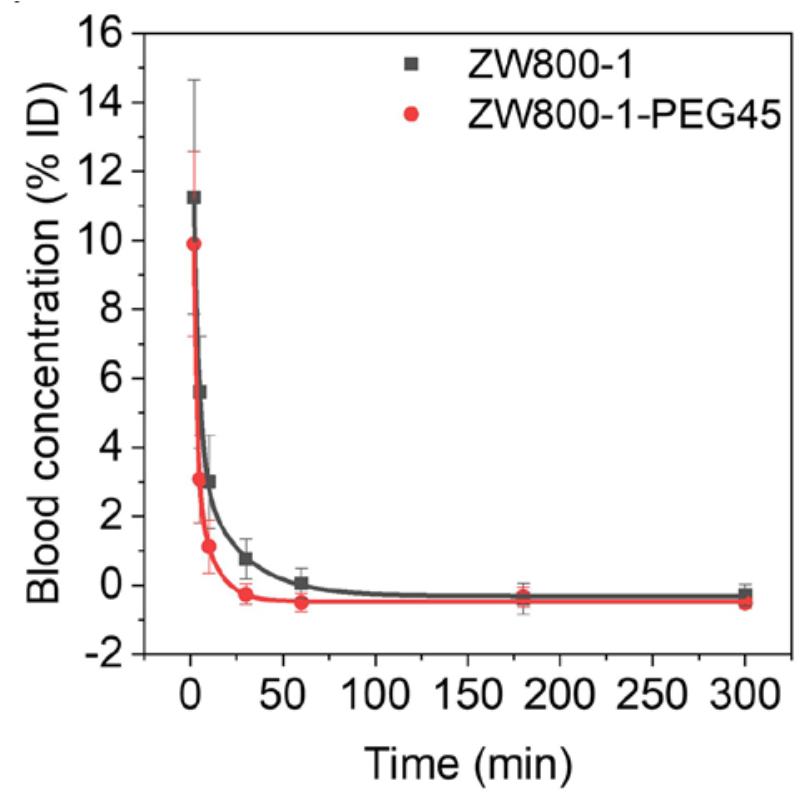

Figure S9. Blood pharmacokinetics of free ZW800-1and ZW800-1-PEG45. n=3

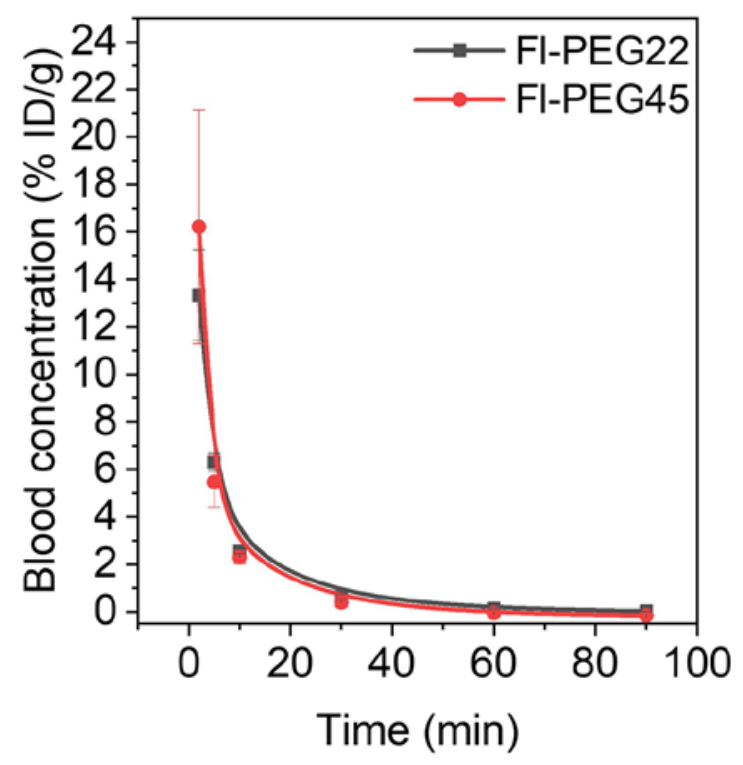

Figure S10. Blood pharmacokinetics of Fl-PEG22 and Fl-PEG45. n=3 


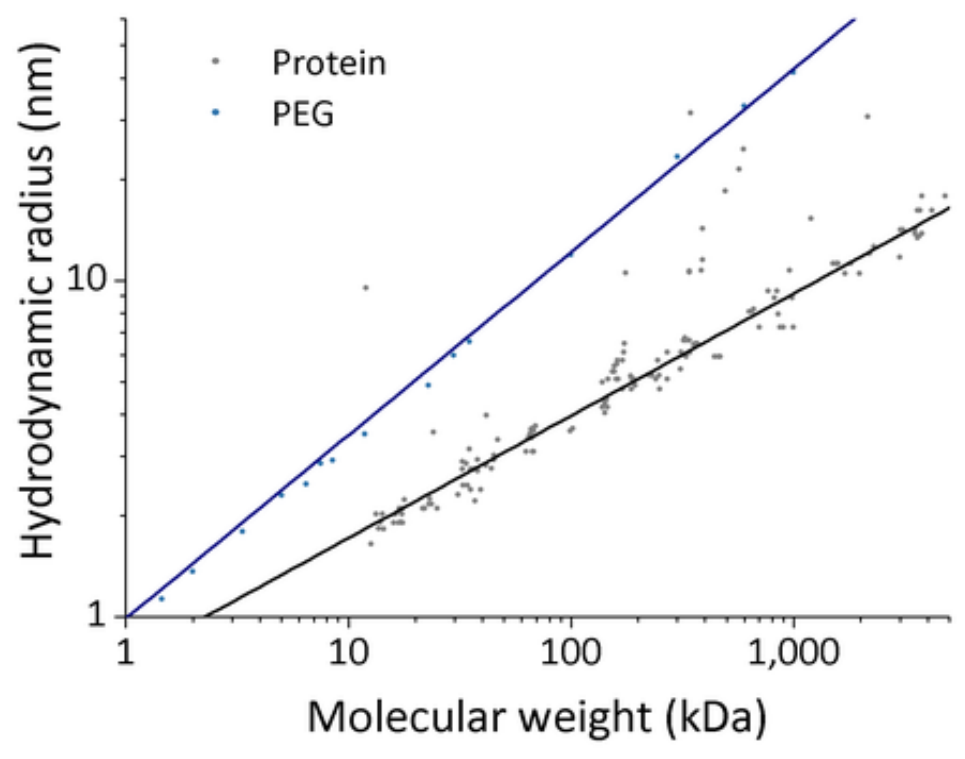

Figure S11. The relationship between molecular weight with hydrodynamic radius of folded molecule (PEG) and unfolded protein ${ }^{1-2}$. (These curves can be directly found online ${ }^{3}$ ) 


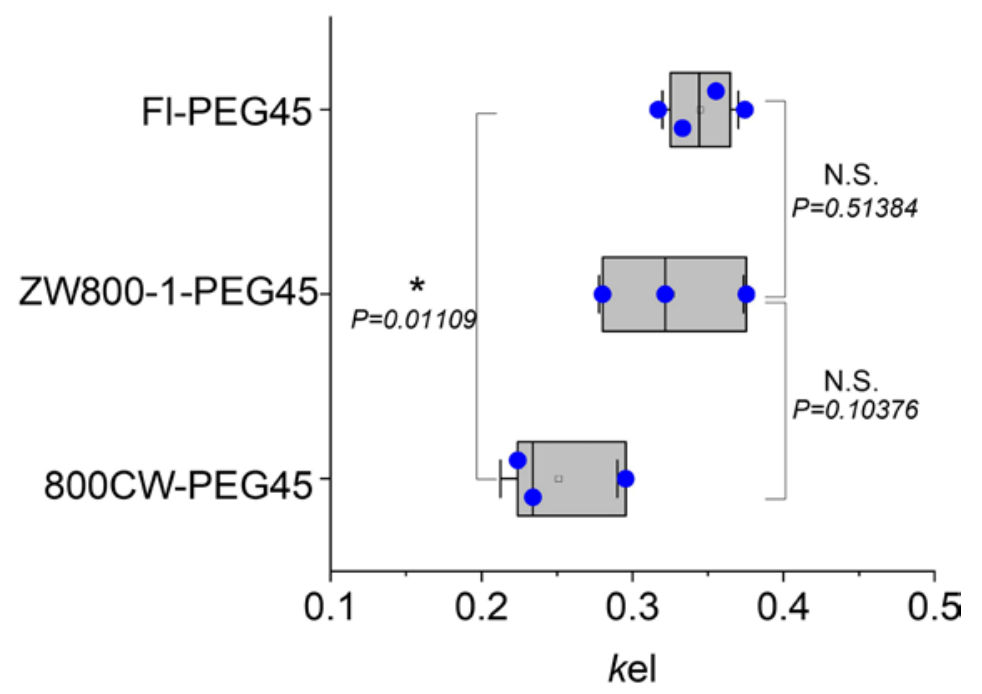

Figure S12. The comparison among 800CW-PEG45, ZW800-1-PEG45 and fluorescinePEG45 in elimination rate constant (kel). N.S. no significant difference based on Student's ttest.

\section{Reference}

1. Tyn, M. T.; Gusek, T. W., Prediction of diffusion coefficients of proteins. Biotechnology and bioengineering 1990, 35 (4), 327-338.

2. $\quad$ Armstrong, J. K.; Wenby, R. B.; Meiselman, H. J.; Fisher, T. C., The hydrodynamic radii of macromolecules and their effect on red blood cell aggregation. Biophysical journal 2004, 87 (6), 4259-4270.

3. $\quad$ https://www.fluidic.com/support/faq/convert-hydrodynamic-radius-to-mw/ 